\title{
Robotic excision of interaortocaval paraganglioma: a case report with the literature review
}

Anoop Handa ${ }^{1,2^{*}} \mathbb{D}$, Sharat Chandra Dash ${ }^{1}$, Nimit Solanki ${ }^{3}$ and Gagandeep Singh ${ }^{4}$

\begin{abstract}
Background: Paragangliomas are rare neuroendocrine tumors of extra adrenal origin. The symptomatic paragangliomas require multimodal treatment approach.

Case Presentation: We report a case of 34-year-old female who presented with complains of headache and blurring of vision. Examination revealed Hypertensive crisis with grade 3 retinopathy. The patient was diagnosed as a case of extra-adrenal paraganglioma during evaluation for secondary hypertension. Magnetic Resonance Imaging of the abdomen revealed $23^{*} 28^{*} 28 \mathrm{~mm}$ mass lesion in the inter-aortocaval region at the level of $L 3-L 4$. The patient was started on medical management for hypertension and was managed safely with robot-assisted surgery, owing to enhanced visualization of feeding vessels and avoidance of excessive manipulation of tumor.
\end{abstract}

Conclusions: Our case report emphasizes on the robot assisted surgical technique for the removal of tumor in a vulnerable anatomical position and presents a review of the literature.

Keywords: Interaortocaval, Paraganglioma, Robotic Surgery

\section{Background}

Extra-adrenal pheochromocytoma are called Paraganglioma (PPGLs). They arise from chromaffin tissue derived from primitive neuroectoderm. Some are asymptomatic and some present with adrenergic symptoms due to release of catecholamines. Symptomatic paraganglioma are usually managed with surgical resection. Minimal invasive techniques are used recently in management of these tumors, we report a case of Interaortocaval paraganglioma managed with Robotic excision.

\section{Case presentation}

34-year-old female presented with complains of headache, blurring of vision and excessive sweating of 4 months duration. She denied any history of vomiting,

\footnotetext{
*Correspondence: dr.anoop10@gmail.com

1 Department of Urology, Army Hospital Research and Referral, New Delhi 110010 , India

Full list of author information is available at the end of the article
}

syncope, chest pain breathlessness, oliguria, altered sensorium, neurological deficit, menstrual irregularity, weight loss or altered bowel habits. There was no significant medical/surgical history in the past. General examination revealed BP-200/130 $\mathrm{mmHg}, \mathrm{PR}-72 /$ min. Fundoscopy-Grade 3 hypertensive retinopathy. Rest systemic examination was unremarkable. Patient was started on antihypertensives and evaluated further as case of secondary hypertension. Baseline hematological, biochemistry workup and thyroid profile were normal. Twenty-four h urinary Metanephrines-1306mcg (normal-<600mcg) and Normetaphrines-1332mcg (normal-23-105 mcg). Doppler of renal vessels was normal. Magnetic Resonance imaging (MRI) abdomen showed $23 * 28 * 28 \mathrm{~mm}$ well defined smoothly marginated mass lesion in the inter Aortocaval region at level of L3-4, abutting 3rd part of duodenum and pancreas. The mass lesion was homogenously hyperintense on T2W1, hypointense on T1W1 and showed peripheral rim enhancement on post contrast images (Fig. 1). 

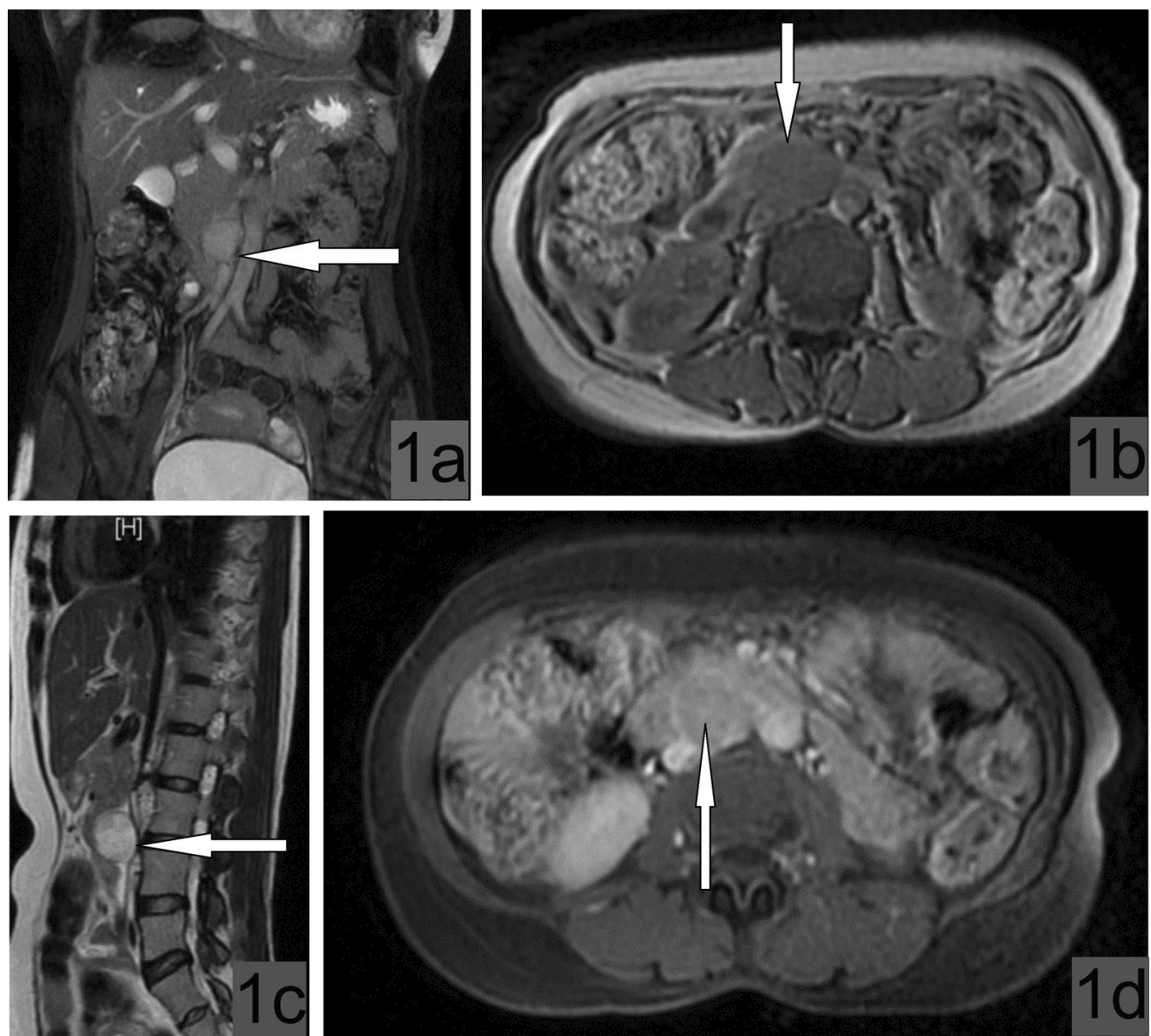

Fig. 1 a Coronal section of Magnetic resonance imaging (MRI) showing $2.3 \times 2.8 \times 2.8 \mathrm{cms}$ well defined smoothly marginated mass lesion in the inter Aortocaval region at level of $L 3-L 4$ vertebra. b Axial section showing lesion is Hypointense on T1 weighted images. c Sagittal section showing lesion homogenously hyperintense on T2 weighted images. $\mathbf{d}$ Axial section showing peripheral rim enhancement and heterogenous central enhancement on post contrast images

${ }^{131}$ I-metaiodobenzylguanidine (MIBG) scan showed faint avidity suggesting tumor of neural crest origin. The diagnosis of interaortocaval paraganglioma was made and subsequently robotic excision was planned. She was started on preoperative preparation with good hydration, started on alpha blockers, Angiotensin-Converting Enzyme inhibitors and calcium channel blockers; beta blockers were started after 1 week of alpha blockers.

\section{Surgery}

Patient was placed in left lateral position under General Anesthesia. Pneumoperitoneum was created, $12 \mathrm{~mm}$ camera port was placed in supra-umbilical location. $5 \mathrm{~mm}$ robotic ports were placed in epigastric, right hypochondrium and right iliac region in spino-umbilical line. 2 assistant ports were placed triangulating the robotic ports for suctioning, irrigation and clip application.
Medial mobilization of right colon done; hepatic flexure was dissected; duodenum was kocherized. Tumor bulge was identified; on further dissection IVC was identified; gonadal vein was inadvertently injured and ligated. The attachments around the tumor were carefully dissected; 2 vein tributaries draining to IVC were identified and clipped. Solitary arterial supply arising from aorta was identified and clipped (Fig. 2) Tumor was extracted into endocatch bag and removed. Intraoperative blood loss was $50 \mathrm{ml}$ and total time of surgery was $150 \mathrm{~min}$. During the procedure, $\mathrm{BP}$ was stable with minimal fluctuations $(150-200 \mathrm{mmHg}$ ) during handling of the tumor were noted. Postoperatively, patient was monitored in intensive care overnight and was shifted to the postoperative ward the following day. Abdominal drain was removed on 2 nd postoperative day and patient was discharged on single antihypertensive. Final histopathology 

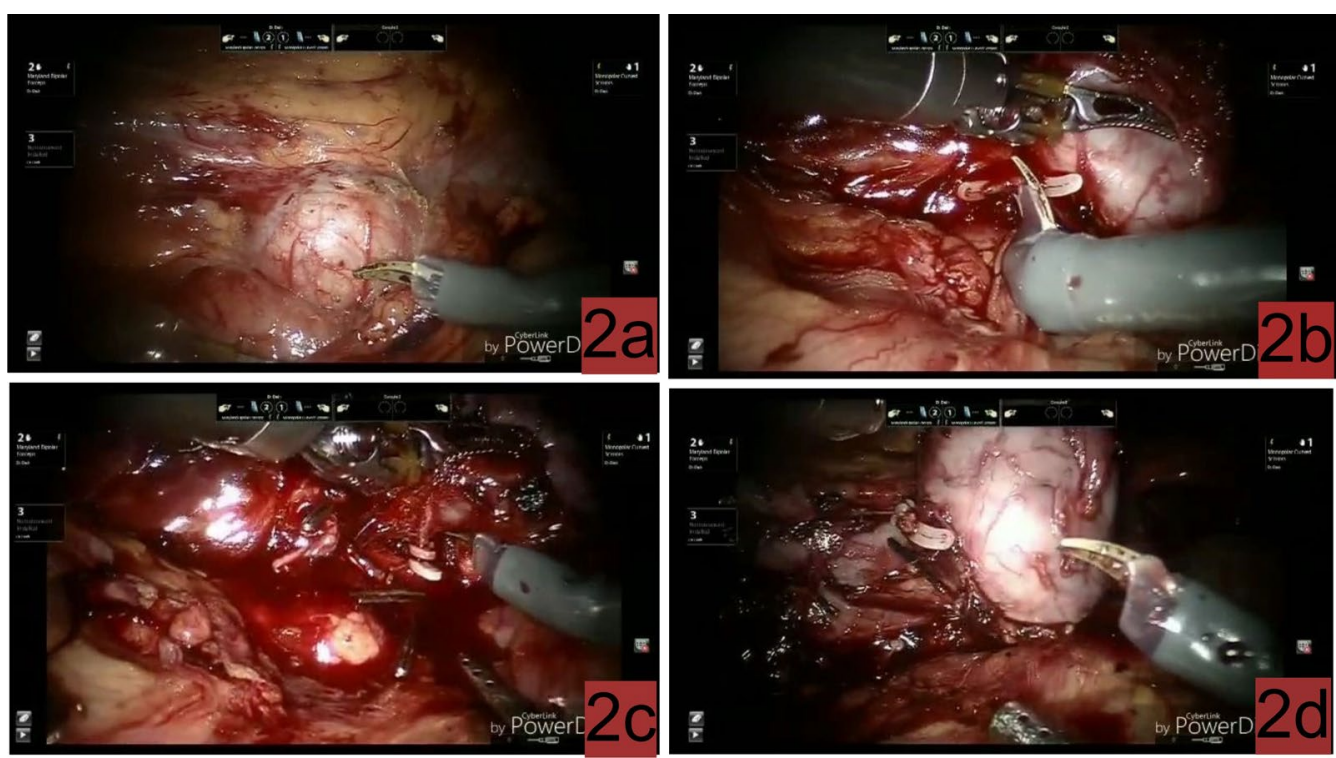

Fig. 2 a Tumor bulge identified after colon mobilization and kocherization of duodenum. b Clipping and division of venous tributary. c Solitary arterial supply clipped. $\mathbf{d}$ Final removal of specimen

showed paraganglioma showing immunopositivity with chromogranin and synaptophysin (Fig. 3). After 1 month, her antihypertensive was stopped and urinary catecholamines showed normal levels. Follow-up after 1 year of surgery, patient was asymptomatic, normotensive with no evidence of relapse or recurrence on MIBG scan and normal urinary catecholamines levels.

\section{Discussion}

Pheochromocytomas are rare, catecholamine-secreting tumor with annual incidence in general population is approximately $0.6-0.8$ cases per 100,000 person-years [1]. It most commonly arises from adrenal medulla, and $1-25 \%$ of the tumors are found in extra-adrenal sites which are termed paraganglioma. The embryological development of paragangliomas occurs from chromaffin cells in the preaortic paravertebral sympathetic plexus that are derivatives of neural crest cells. There are sympathetic paragangliomas that arise from sympathetic tissue and are found in abdominal region, and parasympathetic paraganglioma that arise from parasympathetic tissue and are often found in the thorax, head and neck [2]. The most common sites of paragangliomas include organ of Zuckerkandl located between aortic bifurcation and inferior mesenteric artery, carotid body, head, mediastinum and periaortic region. Majority of paragangliomas are sporadic; however, $30 \%$ are associated with familial syndromes such as Von Hippel lindau, Neurofibromatosis type 1, Multiple Endocrine Neoplasia (MEN) type 2 and carney triad [3].
Paragangliomas are usually classified as functional and nonfunctional. The symptoms of functional paraganglioma are related to catecholamine release, including paroxysmal hypertension, diaphoresis, headache, palpitations and tachycardia. Rarely- Hyperglycemia, panic attacks, fever, weight loss, myocardial infarctions, osteolytic bone metastases, and Raynaud's phenomenon can occur [4]. They occur more often in men and usually in the 3rd or 4th decade of life. $30-40 \%$ of the paragangliomas are malignant; it is difficult to differentiate between benign and malignant histologically and malignancy is defined on basis of metastasis [5].

The diagnosis usually requires biochemical, anatomical and functional imaging. Elevated catecholamines and their metabolites (Metanephrines and Vanillylmandelic Acid) in serum and urine confirm the diagnosis [6]. Anatomical imaging with CT and MRI scans is useful in localizing the tumor. The tumor on CT scan usually appears as hypervascular masses with areas of intralesional hemorrhages and necrosis (salt and pepper appearance) [7]. CT scan have approximately $90 \%$ sensitivity for localization of extra-adrenal tumor, whereas on MRI lesion appear hyperintense on T2 weighted images and have sensitivity approximately 93-100\%. Functional imaging with ${ }^{131}$ I-MIBG scintigraphy carries sensitivity of $60-80 \%$ and specificity of 95-100\%. Recently used imaging techniques include Somatostatin-receptor scintigraphy, DOTATE PET and ${ }^{18} \mathrm{~F}$ FDG PET carries more sensitivity and specificity as compared to MIBG scan [8]. 


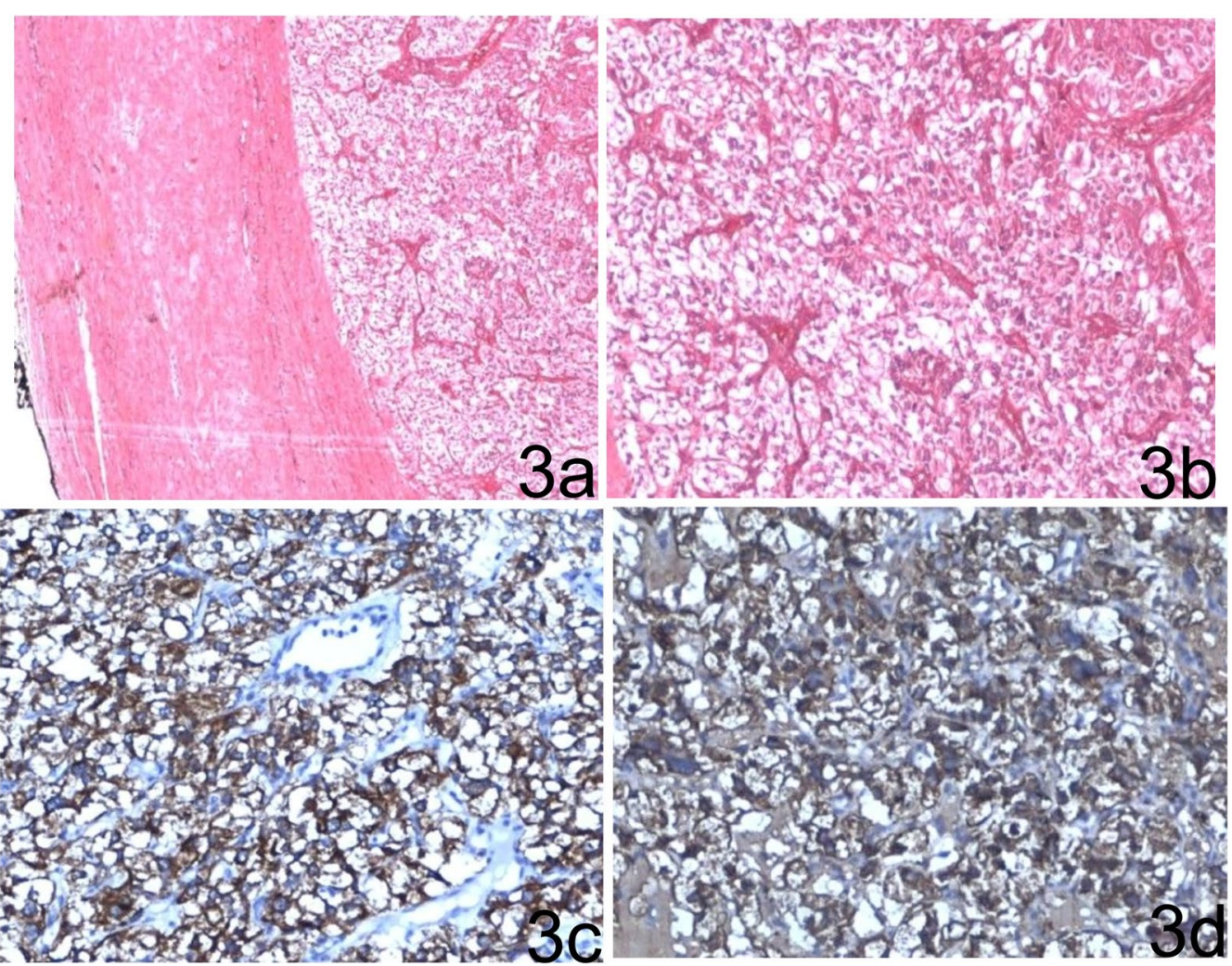

Fig. 3 Microphotographs of $\mathrm{H}$ \& E stained sections of show an encapsulated lesion with underlying zellballen pattern (star) separated by segmented bands of prominent fibrovascular network (a 40x, b 100x). These large polygonal cells have abundant granular eosinophilic cytoplasm and central nuclei with mild nuclear atypia. Tumor cells show cytoplasmic immunopositivity for chromogranin (c) and synaptophysin (d)

The definitive management of paragangliomas is surgical resection. Preoperative stabilization with fluid hydration, alpha blockade and beta blockers is critical [9]. Intraoperative hypertensive crisis during handling of tumor should be discussed with anesthetist and emergency drugs (Sodium Nitroprusside, Nitroglycerine and Esmolol) must be available for rapid control of hypertensive crisis. There are case reports available managing these tumors with minimal invasive techniques such as laparoscopy [10], we preferred robot-assisted technique owing to the difficult location of the tumor. Robotassisted surgery has been used in few cases, there is no standard technique defined due to rarity of this condition $[11,12]$.

\section{Conclusions}

Surgical resection was successfully completed in this case due to sharp dissection, better control, and enhanced visualization provided by robotic instruments, especially in vulnerable anatomical location between IVC and aorta. Robotic surgery offered an opportunity to tackle the enlarged feeding vessels first, improved dexterity and without any manipulation of the tumor leading to better patient outcomes.

\section{Abbreviations \\ MIBG: ${ }^{131}$ I-metaiodobenzylguanidine; MRI: Magnetic resonance imaging; MEN: Multiple endocrine neoplasia; PET: Positron emission tomography; FDG: FluoroDeoxyGlucose.}

\section{Supplementary Information}

The online version contains supplementary material available at https://doi. org/10.1186/s12301-021-00264-5.

Additional file 1. Intraoperative video showing robotic resection of paraganglioma.

\section{Acknowledgements}

Not applicable.

\section{Authors' contributions}

Concept and Study design: AH, SCD. Method and material preparation: NS, GS. Manuscript preparation: AH, GS. Critical Review: SCD, GS, AH. All authors have read and approved the final manuscript.

Funding

This research received no grant from any funding agency or any private sector. 


\section{Availability of data and materials}

The dataset generated during the current study is available from the cor-

responding author on reasonable request.

\section{Declarations}

Ethical approval and consent to participate

Not applicable.

\section{Consent for publication}

Written informed consent was obtained from the patient before publication.

\section{Competing interests}

None.

\section{Author details}

${ }^{1}$ Department of Urology, Army Hospital Research and Referral, New Delhi 110010, India. 'Department of Urology, JJMS, Bahadurgarh, Haryana, India. ${ }^{3}$ Department of Urology, Base Hospital, New Delhi, India. ${ }^{4}$ Department of Urology, Northern Command, Udhampur, India.

Received: 13 August 2021 Accepted: 26 November 2021

Published online: 20 December 2021

\section{References}

1. Berends AMA, Buitenwerf E, de Krijger RR et al (2018) Incidence of pheochromocytoma and sympathetic paraganglioma in the Netherlands: a nationwide study and systematic review. Eur J Intern Med 51:68-73

2. Asa SL, Ezzat S, Mete $O$ (2018) The diagnosis and clinical significance of paragangliomas in unusual locations. J Clin Med 7(9):280

3. Bryant J, Farmer J, Kessler LJ, Townsend RR, Nathanson KL (2003) Pheochromocytoma: the expanding genetic differential diagnosis. J Natl Cancer Inst 95(16):1196-1204

4. Lenders JW, Eisenhofer G, Mannelli M, Pacak K (2005) Phaeochromocytoma. Lancet 366:665-675

5. Roman-Gonzalez A, Jimenez C (2017) Malignant pheochromocytomaparaganglioma: pathogenesis, TNM staging, and current clinical trials. CurrOpin Endocrinol Diabetes Obes 24(3):174-183

6. Eisenhofer G, Lenders JW, Pacak K (2004) Biochemical diagnosis of pheochromocytoma. Front Horm Res 31:76-106

7. Vikas V, Ahmed Z, Singh O, Pal SS, Songra MC. Primary retroperitoneal inter-aorto-caval paraganglioma of the organ of Zuckerkandl. J Clin Diagn Res. 2014;8(7):ND06-7.

8. Han S, Suh CH, Woo S, Kim YJ, Lee JJ (2019) Performance of 68Ga-DOTAconjugated somatostatin receptor-targeting peptide PET in detection of pheochromocytoma and paraganglioma: a systematic review and metaanalysis. J Nucl Med 60:369-376

9. Lenders JW, Duh QY, Eisenhofer G et al (2014) Pheochromocytoma and paraganglioma: an endocrine society clinical practice guideline. J Clin Endocrinol Metab 99(6):1915-1942

10. Kravarusic D, Pinto-Rojas A, Al-Assiri A, Sigalet D (2007) Laparoscopic resection of extra-adrenal pheochromocytoma-case report and review of the literature in pediatric patients. J Pediatr Surg 42(10):1780-1784

11. Cochetti G, Cottini E, Barillaro F, Lepri E, Boni A, Pohja S et al (2014) Robot assisted laparoscopic excision of a paraganglioma: new therapeutic approach. Int. Braz J Urol. 40(2):279-80

12. Lehrfeld T, Natale R, Sharma S, Mendoza PJ, Schwab li CW, Lee DI (2010) Robot-assisted excision of a retroperitoneal mass between the left renal artery and vein. JSLS 14(3):447-449

\section{Publisher's Note}

Springer Nature remains neutral with regard to jurisdictional claims in published maps and institutional affiliations.

\section{Submit your manuscript to a SpringerOpen ${ }^{\circ}$ journal and benefit from:}

- Convenient online submission

- Rigorous peer review

- Open access: articles freely available online

- High visibility within the field

- Retaining the copyright to your article 